\title{
Entransy decrease principle of heat transfer in an isolated system
}

\author{
CHENG XueTao, LIANG XinGang* \& GUO ZengYuan \\ Key Laboratory for Thermal Science and Power Engineering of Ministry of Education, School of Aerospace, Tsinghua University, Beijing 100084, \\ China
}

Received July 28, 2010; accepted September 29, 2010

\begin{abstract}
The entropy increase principle for an isolated system and the criteria of thermal equilibrium for an isolated system and systems with prescribed temperature and volume can be derived on the basis of the concept of entropy and the first and second laws of thermodynamics. In this paper, the entransy decrease principle for an isolated system is introduced on the basis of the concept of entransy. It is found that the entransy of an isolated system always decreases during heat transfer. This principle can be taken as an expression of the second law of thermodynamics for heat transfer. The thermal equilibrium criteria for an isolated system and a closed system are also introduced. It is found that when an isolated system reaches thermal equilibrium, its entransy is a minimum value. This criterion is referred to as the minimum entransy principle. When a closed system reaches thermal equilibrium, its free entransy is also a minimum value. This criterion is referred to as the minimum free entransy principle. Therefore, like entropy, entransy can be considered an arrow of time in heat transfer and used to describe the thermal equilibrium state.
\end{abstract}

entropy increase principle, thermal equilibrium criterion, entransy decrease principle, minimum entransy principle, minimum free entransy principle

Citation: Cheng X T, Liang X G, Guo Z Y. Entransy decrease principle of heat transfer in an isolated system. Chinese Sci Bull, 2011, 56: 847-854, doi: $10.1007 / \mathrm{s} 11434-010-4328-4$

Irreversibility is a common characteristic of all physical processes in nature. For instance, in friction processes, mechanical work can be totally transformed into heat. However, heat cannot totally be turned into mechanical work automatically. In diffusion processes, two kinds of fluid in a mixture cannot separate from each other automatically. A reversible physical process is the ideal case in which there is no dissipation, and this cannot be achieved in practice [1]. Therefore, for any physical process, there is always an evolution direction that can be described by the second law of thermodynamics. In 1850, Clausius described the law with an expression that it is impossible to construct a device that operates in a cycle and produces no effect other than the transfer of heat from a lower-temperature body to a highertemperature body [1]. In 1851, Kelvin introduced another expression based on heat-work conversion; that is, it is impossible for any device to operate in a cycle and receive heat

*Corresponding author (email: liangxg@tsinghua.edu.cn) from a single reservoir and produce an equivalent amount of mechanical work [1]. The Clausius expression and Kelvin expression have been proved to be equivalent [1].

Clausius further introduced a quantitative description of the irreversible process using a state function, entropy, when he investigated the Carnot cycle in 1854 . The expression of entropy is $[1,2]$

$$
\mathrm{d} S=\left(\frac{\delta Q}{T}\right)_{\mathrm{rev}},
$$

where $\mathrm{d} S$ is the variation of entropy, $\delta Q$ is the heat exchange between the system and the environment, and $T$ is the temperature of the heat source, which is also the environment temperature for the reversible process. The subscript 'rev' indicates that the process is an ideal reversible process. Clausius derived the Clausius inequality for entropy, which expresses the second law of thermodynamics with a mathematical expression and makes it possible to 
calculate the irreversibility as a quantity. Since then, researchers gradually developed the thermal equilibrium criteria for various systems on the basis of the concept of entropy; e.g. the isolated system, the system at constant temperature and volume, and the system at constant temperature and pressure [1]. With these fundamental and important developments, the concept of entropy is widely used in thermodynamics, cybernetics, probability theory, life sciences and other academic fields.

The application of entropy is also researched in the field of heat transfer. From the view of thermodynamics, the heat transfer processes are irreversible and in non-equilibrium. For non-equilibrium thermodynamic processes, Onsager $[3,4]$ established fundamental equations and derived the principle of the least dissipation of energy employing variational theory. On the basis of the idea that the entropy generation of a thermal system at steady state should be a minimum, Prigogine [5] developed the principle of minimum entropy generation. Furthermore, Bejan [6-9] developed expressions of entropy generation for heat and fluid flows and then introduced the principle of least entropy generation to the heat-transfer optimizations. Many research groups have done much work on heat transfer optimization using the concept of entropy; e.g. Poulikakos and Bejan [10], Erek and Dincer [11], Shah and Skiepko [12]. Their work showed that entropy can describe the irreversibility of heat transfer effectively.

In the history of sciences, some principles have had more than one expression. In thermodynamics, for instance, the Clausius expression and Kelvin expression both describe the second law even though the expressions are different. In quantum mechanics, the Schrodinger equation and matrix mechanics reveal the quantum world in different ways.

One may then ask whether entropy is the only concept that can be applied to describe the irreversibility of heat transfer. Before we answer this question, let us introduce a new concept, entransy [13], which was proposed by Guo et al. [13] to describe the potential energy of heat transfer by comparing electricity and heat transfer. Entransy was initially referred to as the heat transport potential capacity $[14,15]$. If a body has an internal energy $U$ and temperature $T$, its entransy is defined as

$$
G=\frac{1}{2} U T .
$$

Guo et al. [13] established the concept of entransy flux. They postulated that the entransy of a body represents its heat transport ability. This capacity does not only depend on the body's temperature but also on its internal heat. The heat transport ability decreases when heat is transferred from a high-temperature body to a low-temperature body and entransy is dissipated. Employing the concept of entransy dissipation, they developed the principle of extremum entransy dissipation and the principle of minimum thermal resistance. In recent years, these principles have been applied to optimize or enhance heat conduction [13,16-21], heat convection [13,22,23] and thermal radiation $[24,25]$ and determine the optimal designs of heat exchangers [26-29].

Eq. (2) tells us that entransy is half the product of the internal energy and temperature of the system for a heat transfer process that does not involve heat-work conversion. We know that both the internal energy and temperature are state quantities. Therefore, like entropy, entransy is also a state quantity. Entropy can describe the irreversibility of heat transfer and be used to establish thermal equilibrium criteria for certain systems. This paper attempts to determine whether entransy can describe irreversibility and be used to establish thermal equilibrium criteria.

\section{Entropy increase principle and its thermal equilibrium criteria [1]}

Using the definition of entropy (eq. (1)), we investigate the thermodynamic cycle that is shown in Figure 1 and has $n$ heat sources. The temperature of the $i$ th heat source is $T_{i}$. The system $\Sigma$ comes into contact with each heat source in turn. Finally, it returns to the first source and the cycle is complete.

We assume that system $\Sigma$ receives energy $Q_{i}$ from the $i$ th heat source and outputs mechanical work $W$. According to the first law of thermodynamics,

$$
\sum_{i=1}^{n} Q_{i}=W .
$$

We consider an auxiliary heat source whose temperature is $T_{0}$, and $n$ Carnot engines working between the heat sources and the auxiliary heat source. We assume that the $i$ th engine receives energy $Q_{i 0}$ from the auxiliary heat source and that the energy $Q_{i}{ }^{\prime}$ that the $i$ th heat source obtains from the $i$ th engine is equal to the energy $Q_{i}$ that system $\Sigma$ receives from the $i$ th heat source. According to the first law of thermodynamics, the output mechanical work of all the engines is

$$
W_{0}=\sum_{i=1}^{n} Q_{i 0}-\sum_{i=1}^{n} Q_{i}^{\prime}=Q_{0}-W,
$$

where $Q_{0}$ is the total energy that the Carnot engines receive from the auxiliary heat source and $W$ is the output work of system $\Sigma$. We thus obtain

$$
W_{0}+W=Q_{0} .
$$

If system $\Sigma$ and all the Carnot engines are treated as a large system, then the total system finishes its cycle while system $\Sigma$ and the Carnot engines finish their cycles. $Q_{0}$ is then the energy that the total system receives from the environment, and it is only from one auxiliary heat source. 


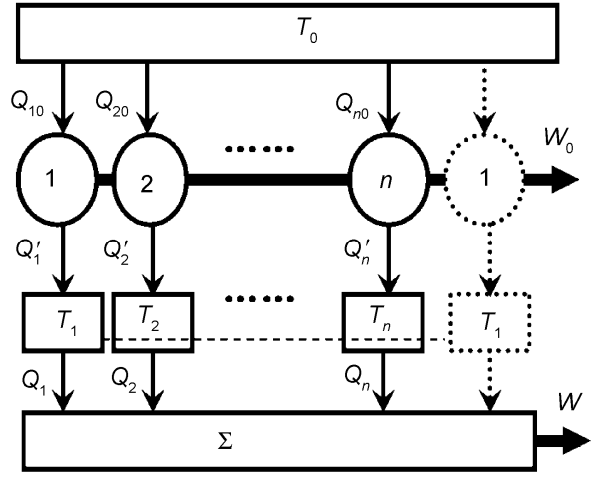

Figure 1 Sketch of one thermodynamic cycle.

Considering the Kelvin expression of the second law of thermodynamics and eq. (5), we obtain

$$
W_{0}+W=Q_{0} \leqslant 0 .
$$

In each Carnot cycle,

$$
\frac{Q_{i 0}}{T_{0}}=\frac{Q_{i}^{\prime}}{T_{i}} .
$$

As $Q_{i}^{\prime}$ is equal to $Q_{i}$,

$$
Q_{0}=\sum_{i=1}^{n} Q_{i 0}=T_{0} \sum_{i=1}^{n} \frac{Q_{i}}{T_{i}}
$$

Considering eq. (6) and that $T_{0}$ is positive,

$$
\sum_{i=1}^{n} \frac{Q_{i}}{T_{i}} \leqslant 0
$$

We know that the Carnot cycle is reversible. Therefore, if the energy $Q_{i}$ in eq. (9) is replaced by its opposite value, the equation remains tenable. Thus,

$$
\sum_{i=1}^{n} \frac{-Q_{i}}{T_{i}} \leqslant 0
$$

From eqs. (9) and (10), we obtain

$$
\sum_{i=1}^{n} \frac{Q_{i}}{T_{i}}=0 .
$$

If the number of heat sources is infinite, and the temperature differences of the adjacent heat sources are infinitesimal, eq. (9) can be rewritten as

$$
\oint \frac{\delta Q}{T} \leqslant 0
$$

This is the Clausius inequality. The equal sign in eq. (12) is tenable only when the process is reversible, while the sign of "less than" is tenable for any irreversible process. According to the definition of eq. (1), we obtain

$$
\mathrm{d} S \geqslant 0 \text {. }
$$

This is the entropy increase principle, which indicates that the entropy of an isolated system will always increase. Therefore, entropy can be treated as an arrow of time, which gives an evolution direction for any physical process in an isolated system [2].

The thermal equilibrium criterion of the isolated system with constant volume and internal energy can be established according to the entropy increase principle. When the system reaches its thermal equilibrium state, the entropy of the system is a maximum, and

$$
\mathrm{d} S=0 \text {. }
$$

For system $\Sigma$ with prescribed temperature and volume, the thermal equilibrium criterion can also be established. We assume that system $\Sigma$ is in a much larger system $\Sigma^{\prime}$. As system $\Sigma^{\prime}$ is much larger than system $\Sigma$, the heat exchange between the systems has no effect on the temperature $T$ of system $\Sigma^{\prime}$. If system $\Sigma^{\prime}$ and $\Sigma$ are treated as one, this new system is surely an isolated system. According to eq. (14),

$$
\mathrm{d} S_{0}=\mathrm{d}\left(S+S^{\prime}\right)=\mathrm{d} S+\mathrm{d} S^{\prime}=0,
$$

when this new system reaches thermal equilibrium. We assume that the change in the internal energy of system $\Sigma$ is $\mathrm{d} U$, which can only result from system $\Sigma^{\prime}$. The entropy change of system $\Sigma^{\prime}$ can then be expressed as

$$
\mathrm{d} S^{\prime}=-\frac{\mathrm{d} U}{T} .
$$

Considering that $T$ is given, Substituting eq. (16) into eq. (15) leads to

$$
\mathrm{d} F=0,
$$

where $F$ is the Helmholtz free energy, whose expression is

$$
F=U-T S \text {. }
$$

For system $\Sigma$ with prescribed temperature and volume, eqs. (17) and (18) tell us that the Helmholtz free energy is a minimum when the system is in thermal equilibrium. This is the thermal equilibrium criterion of the system with prescribed temperature and volume.

The entropy increase principle and its thermal equilibrium criteria are restated above on the basis of the concept of entropy and the first and second laws of thermodynamics. With these conclusions, the irreversibility of any physical process can be quantitatively calculated, and whether one isolated system or one system with prescribed temperature and volume is in thermal equilibrium can be judged. Corresponding to the principle and thermal equilibrium criteria described above, similar principles can be established for heat transfer on the basis of the concept of entransy.

\section{Entransy decrease principle of heat transfer in an isolated system}

A common heat transfer process as shown in Figure 2 is 


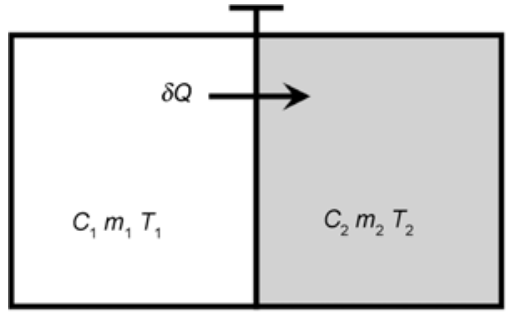

Figure 2 Sketch of heat transfer in an isolated system.

investigated so as to determine if entransy can indicate the evolution direction of heat transfer. The figure shows that the isolated system is composed of two subsystems. The volumes of the subsystems and the internal energy of the system are invariant. The system and its subsystems are infinite from a microscopic point of view, and we can define the heat capacity, temperature and other parameters of the systems. The heat capacity, mass and temperature of subsystem 1 are $c_{1}, m_{1}$ and $T_{1}$, respectively, while those of subsystem 2 are $c_{2}, m_{2}$ and $T_{2}$. There is a plate with ideal heat insulation between the subsystems. We assume that there is no energy transfer between the subsystems before the plate is taken away. In an instant, the plate is removed and there is then heat transfer between the subsystems. For this kind of system, Han and Guo [30] proved that the entransy of the system decreases before and after the thermal equilibrium state is reached. We analyze the entransy change of the system when a small amount of heat is transferred between the subsystems hereafter.

We consider the relationship between the internal energy, temperature and heat capacity of the system, $U=\mathrm{cm} T$. The entransy of the subsystems can be obtained from eq. (2):

$$
\begin{aligned}
G_{1} & =\frac{1}{2} c_{1} m_{1} T_{1}^{2}, \\
G_{2} & =\frac{1}{2} c_{2} m_{2} T_{2}^{2} .
\end{aligned}
$$

We suppose $\delta Q$ is transferred from subsystem 1 to 2 in a period of time after the ideal heat insulation plate is removed; then

$$
\delta Q=\mathrm{d} U+\delta W
$$

As the volumes of the subsystems do not change, $\delta W$ is zero. The heat transfer between the subsystems can only affect the internal energy of the subsystems. Considering $U$ $=c m T$, the temperatures of the subsystems after $\delta Q$ is transferred can be expressed as

$$
\begin{gathered}
T_{1}^{\prime}=\left(c_{1} m_{1} T_{1}-\delta Q\right) / c_{1} m_{1}, \\
T_{2}^{\prime}=\left(c_{2} m_{2} T_{2}+\delta Q\right) / c_{2} m_{2} .
\end{gathered}
$$

According to eq. (2), the entransy of the subsystems is then

$$
G_{1}^{\prime}=\frac{1}{2} c_{1} m_{1} T_{1}^{\prime 2}=\frac{1}{2} c_{1} m_{1}\left(T_{1}-\delta Q / c_{1} m_{1}\right)^{2},
$$

$$
G_{2}^{\prime}=\frac{1}{2} c_{2} m_{2} T_{2}^{\prime 2}=\frac{1}{2} c_{2} m_{2}\left(T_{2}+\delta Q / c_{2} m_{2}\right)^{2}
$$

The entransy change of the whole system is

$$
\mathrm{d} G=\left(G_{1}^{\prime}+G_{2}^{\prime}\right)-\left(G_{1}+G_{2}\right) .
$$

Substituting eqs. (19), (20), (24) and (25) into eq. (26) gives

$$
\mathrm{d} G=\frac{1}{2} \delta Q\left[\kappa \delta Q+2\left(T_{2}-T_{1}\right)\right]
$$

where $\kappa=1 / c_{1} m_{1}+1 / c_{2} m_{2}$.

If $\delta Q>0$, according to the Clausius expression of the second law of thermodynamics, it is required that

$$
T_{1}^{\prime} \geqslant T_{2}^{\prime}
$$

Substituting eqs. (22) and (23) into eq. (28) gives

$$
\begin{aligned}
& \kappa \delta Q+T_{2}-T_{1} \leqslant 0, \\
& T_{2}-T_{1} \leqslant-\kappa \delta Q<0 .
\end{aligned}
$$

Substituting eqs. (29) and (30) into eq. (27) and considering $\delta Q>0$ yield

$$
\mathrm{d} G<0 .
$$

Let us consider an ideal heat transfer process in which the temperature difference of the subsystems is infinitesimal and the amount of heat transported has no effect on the temperatures of the subsystems. It is required that $\kappa=0$ and $T_{1}=T_{2}$. Therefore,

$$
\kappa \delta Q+2\left(T_{2}-T_{1}\right)=0 .
$$

Substituting eq. (32) into eq. (26) gives

$$
\mathrm{d} G=0 .
$$

From eqs. (31) and (33), the variation in entransy is

$$
\mathrm{d} G \leqslant 0 .
$$

Eq. (34) tells us that the entransy of an isolated system never increases during heat transfer. The equal sign is tenable only when the process is an ideal heat transfer process with infinitesimal temperature difference, while the sign of "less than" is tenable for any heat transfer process in practice. This is the entransy decrease principle of heat transfer in an isolated system.

As the physical parameter that describes the heat transfer ability, entransy is found always to decrease during the process of heat transfer in an isolated system. The irreversibility of heat transfer processes is because of the loss of entransy or heat transfer ability. Once heat reaches a low-temperature body, it can never return to a hightemperature body. Similar to the entropy increase principle, the entransy decrease principle gives an evolution direction for any heat transfer process; that is, the entransy of an 
isolated system always decreases. Therefore, it is also a time arrow for heat transfer. This principle can be considered an expression of the second law of thermodynamics for heat transfer.

\section{Thermal equilibrium criteria based on the entransy decrease principle}

\subsection{Thermal equilibrium criterion of the isolated sys- tem: the minimum entransy principle}

Similar to the thermal equilibrium criterion of the isolated system based on the concept of entropy, we can also establish a new thermal equilibrium criterion for the isolated system with prescribed internal energy and volume based on the concept of entransy.

For any isolated system, the temperature of any part of the system should be the same and not change after the system is in thermal equilibrium [1].

As shown in Figure 3, the isolated system is composed of $n$ parts. Initially, the heat capacity, temperature and mass of the $i$ th part are $c_{i}, T_{i}$ and $m_{i}$, respectively. The initial entransy of the system is the sum of each part:

$$
G_{0}=\sum_{i=1}^{n} \frac{1}{2} m_{i} c_{i} T_{i}^{2}
$$

Heat is transferred if there are initially temperature differences among parts of the system. After a period $t$, the internal energy of the $i$ th part has changed by $\Delta U_{i}$, and the temperature of the $i$ th part is

$$
T_{i-t}=T_{i}+\Delta U_{i} / m_{i} c_{i} .
$$

According to the first law of thermodynamics,

$$
\sum_{i=1}^{n} \Delta U_{i}=0
$$

The entransy of the system is

$$
G=\sum_{i=1}^{n} \frac{1}{2} m_{i} c_{i} T_{i-t}^{2}=\sum_{i=1}^{n} \frac{1}{2} m_{i} c_{i}\left(T_{i}+\Delta U_{i} / m_{i} c_{i}\right)^{2}
$$

on the basis of eq. (36). Therefore, the entransy decrease of the system from its initial state can be expressed as

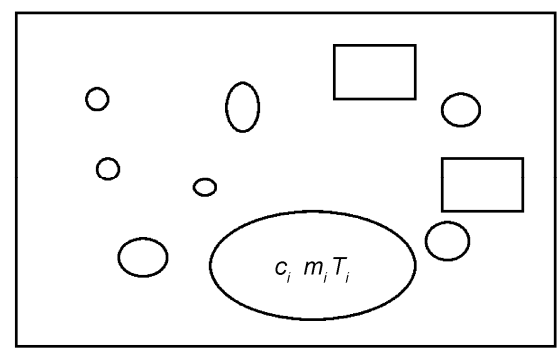

Figure 3 Sketch of an isolated system.

$$
G_{\mathrm{dec}}=G_{0}-G=-\sum_{i=1}^{n}\left[T_{i} \Delta U_{i}+\left(\Delta U_{i}\right)^{2} / 2 m_{i} c_{i}\right] .
$$

To find the extreme value of eq. (39) with the limiting condition of eq. (37), a functional is established as

$$
\Pi=-\sum_{i=1}^{n}\left[T_{i} \Delta U_{i}+\left(\Delta U_{i}\right)^{2} / 2 m_{i} c_{i}\right]+\lambda \sum_{i=1}^{n} \Delta U_{i},
$$

where $\lambda$ is the Lagrange multiplier. Letting the derivative of eq. (40) equal zero, we have

$$
\frac{\partial \prod}{\partial\left(\Delta U_{i}\right)}=-\left(T_{i}+\Delta U_{i} / m_{i} c_{i}\right)+\lambda=0 .
$$

Therefore,

$$
\Delta U_{i}=m_{i} c_{i}\left(\lambda-T_{i}\right)
$$

Substituting eq. (42) into eq. (37) gives

$$
\lambda=\sum_{i=1}^{n} m_{i} c_{i} T_{i} / \sum_{i=1}^{n} m_{i} c_{i}
$$

Combining eqs. (36) and (42), we get

$$
T_{i-t}=\lambda=\sum_{i=1}^{n} m_{i} c_{i} T_{i} / \sum_{i=1}^{n} m_{i} c_{i} .
$$

The above equation implies that the temperatures of different parts of the system are the same (the system reaches thermal equilibrium) when the entransy of the system reaches its extremum value. From eq. (41), we get

$$
\frac{\partial^{2} \prod}{\partial\left(\Delta U_{i}\right)^{2}}=-\frac{1}{m_{i} c_{i}}<0 \text {. }
$$

Therefore, when eq. (44) is justifiable, we find that

$$
\mathrm{d} G_{\mathrm{dec}}=0 .
$$

Moreover, $G_{\mathrm{dec}}$ is a maximum. Thus,

$$
\mathrm{d} G=\mathrm{d}\left(G_{0}-G_{\mathrm{dec}}\right)=-\mathrm{d} G_{\mathrm{dec}}=0 .
$$

The entransy of the system is a minimum.

The entransy decrease principle tells us that heat transfer decreases the entransy of the isolated system. On the basis of this principle, we find that the entransy is a minimum when the isolated system reaches thermal equilibrium. This is the thermal equilibrium criterion of the isolated system. It can also be referred to as the minimum entransy principle.

\subsection{Thermal equilibrium criterion of the closed system: the minimum free entransy principle}

As shown in Figure 4, system $\Sigma$ is a closed system. The volumes of systems $\Sigma$ and $\Sigma^{\prime}$ are given, and system $\Sigma^{\prime}$ is much bigger than $\Sigma$. If systems $\Sigma$ and $\Sigma^{\prime}$ are treated as one system $\Sigma_{0}\left(\Sigma_{0}=\Sigma+\Sigma^{\prime}\right)$, the whole system $\left(\Sigma_{0}\right)$ is isolated. If 


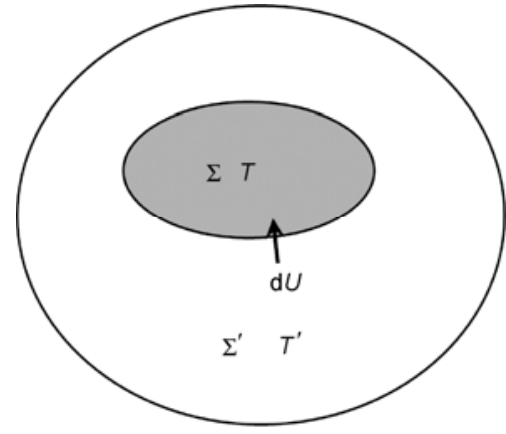

Figure 4 Sketch of the closed system.

we assume that system $\Sigma^{\prime}$ has already reached thermal equilibrium, then its temperature, mass and heat capacity are $T^{\prime}$, $m^{\prime}$ ( $m^{\prime}$ is infinitely large) and $c^{\prime}$, respectively.

As system $\Sigma^{\prime}$ is much bigger than $\Sigma$, the heat transfer between the systems has a negligible effect on $\Sigma^{\prime}$ and $T^{\prime}$ does not change. The minimum entransy principle can be applied to $\Sigma_{0}$ because it is an isolated system. At thermal equilibrium, we have

$$
\mathrm{d} G_{0}=\mathrm{d}\left(G+G^{\prime}\right)=\mathrm{d} G+\mathrm{d} G^{\prime}=0 .
$$

There is no mechanical work for any subsystem as the volumes of $\Sigma$ and $\Sigma^{\prime}$ are given. The heat transfer between the systems can then only affect their internal energy. We assume that the internal energy change of $\Sigma$ is $\mathrm{d} U$. This change can only come from system $\Sigma^{\prime}$. From eq. (2), the entransy change of system $\Sigma^{\prime}$ is

$$
\begin{aligned}
\mathrm{d} G^{\prime} & =\frac{1}{2} c^{\prime} m^{\prime}\left(T^{\prime}-\mathrm{d} U / c^{\prime} m^{\prime}\right)^{2}-\frac{1}{2} c^{\prime} m^{\prime} T^{\prime 2} \\
& =-T^{\prime} \mathrm{d} U+(\mathrm{d} U)^{2} / 2 c^{\prime} m^{\prime} .
\end{aligned}
$$

Recalling that $m^{\prime}$ is infinitely large, we can drop the second term in the above equation, giving

$$
\mathrm{d} G^{\prime}=-T^{\prime} \mathrm{d} U .
$$

Substituting eq. (50) into eq. (48) gives

$$
\mathrm{d} G_{0}=\mathrm{d} G-T^{\prime} \mathrm{d} U=0 .
$$

Considering that $T^{\prime}$ is a constant, we have

$$
\mathrm{d}\left(G-U T^{\prime}\right)=0,
$$

where $G$ and $U$ are the entransy and internal energy for system $\Sigma$, respectively. It is found from eqs. (49) and (50) that $U T^{\prime}$ is the entransy increase for system $\Sigma^{\prime}$ when all the internal energy of $\Sigma$ is transferred to system $\Sigma^{\prime}$. In other words, $G$ and $U T^{\prime}$ represent the entransy for internal energy $U$ when it is in system $\Sigma$ and $\Sigma^{\prime}$ respectively. Corresponding to the definition of the free energy, we can define the free entransy for system $\Sigma$ as

$$
G_{\mathrm{F}}=G-U T^{\prime} .
$$

For the closed system at thermal equilibrium, we have

$$
\mathrm{d} G_{\mathrm{F}}=0 .
$$

From eq. (2) and $U=c m T$, it is noted that eq. (53) is a quadratic function of the temperature. Therefore, the value in eq. (53) is a minimum when eq. (54) holds true. The free entransy is a minimum when the closed system reaches thermal equilibrium. This is the thermal equilibrium criterion for the closed system. It can also be referred to as the minimum free entransy principle.

In [1], the thermal equilibrium criterion of the system with prescribed temperature and pressure is introduced on the basis of the entropy increase principle. However, the volume of that kind of system changes. This relates to mechanical work in the process. This paper only focuses on the heat transfer process without heat-work conversion, and thus, the thermal equilibrium criterion of the system with prescribed temperature and pressure is not discussed here.

\section{Verification of the entransy decrease princi- ple and its thermal equilibrium criteria}

Let us consider the process of heat conduction in a simple isolated system as shown in Figure 5. The heat capacity, mass and temperature of the high-temperature solid body are $c_{\mathrm{H}}, m_{\mathrm{H}}$ and $T_{\mathrm{H}}$, respectively, while those of the lowtemperature solid body are $c_{\mathrm{L}}, m_{\mathrm{L}}$ and $T_{\mathrm{L}}$, respectively. The contact area of the two bodies is $A$. The thermal resistance of the contact area is $R$.

Assuming that the temperature of each body can be calculated with the lumped parameter method, the control equations of the heat transfer process can be expressed as

$$
c_{\mathrm{H}} m_{\mathrm{H}} \frac{\partial T_{\mathrm{H}}}{\partial \tau}=A \frac{T_{\mathrm{L}}-T_{\mathrm{H}}}{R} \text { and } c_{\mathrm{L}} m_{\mathrm{L}} \frac{\partial T_{\mathrm{L}}}{\partial \tau}=A \frac{T_{\mathrm{H}}-T_{\mathrm{L}}}{R} \text {, }
$$

where $\tau$ is time. We can simplify eq. (55) as

$$
\frac{\partial T_{\mathrm{H}}}{\partial \tau}=-k_{\mathrm{H}}\left(T_{\mathrm{H}}-T_{\mathrm{L}}\right) \text { and } \frac{\partial T_{\mathrm{L}}}{\partial \tau}=-k_{\mathrm{L}}\left(T_{\mathrm{L}}-T_{\mathrm{H}}\right),
$$

where $k_{\mathrm{H}}=A / c_{\mathrm{H}} m_{\mathrm{H}} R$ and $k_{\mathrm{L}}=A / c_{\mathrm{L}} m_{\mathrm{L}} R$. If we take both bodies as copper, then $c_{\mathrm{H}}=c_{\mathrm{L}}=386 \mathrm{~J} \mathrm{~kg}^{-1} \mathrm{~K}^{-1}, m_{\mathrm{H}}=m_{\mathrm{L}}=1 \mathrm{~kg}$, and $k_{\mathrm{H}}=k_{\mathrm{L}}=10^{-2} \mathrm{~s}^{-1}$. The initial temperatures of the two

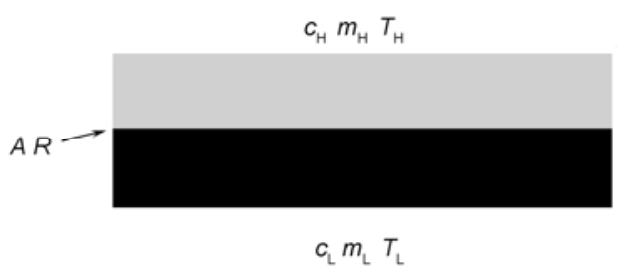

Figure 5 Heat transfer process in a simple isolated system. 
bodies are $T_{\mathrm{H} 0}=300 \mathrm{~K}$ and $T_{\mathrm{L} 0}=280 \mathrm{~K}$. The temperature variations of the two bodies can then be obtained solving eq. (56). The results are shown in Figure 6. The entransy and entropy changes of the system with time are also obtained as shown in Figure 7, in which the entransy is calculated with eq. (2) while the entropy change is calculated with eq. (1).

Figure 6 shows that the temperatures of the two bodies gradually become $290 \mathrm{~K}$, which is the thermal equilibrium temperature of the system. In Figure 7, the entropy of the system increases gradually and has an asymptotic value, which indicates that entropy can give the evolution direction of the heat transfer process and be a measure of the irreversibility. In addition, the asymptotic value of the entropy change shows that the thermal equilibrium criterion of the isolated system based on the concept of entropy, $\mathrm{d} S=0$, describes the thermal equilibrium state effectively. On the other hand, the entransy of the system decreases gradually, but it also has an asymptotic value. Similar to entropy, it is concluded that entransy can be used to give the evolution direction of the process and to measure the irreversibility. The asymptotic value of the entransy also shows that the thermal equilibrium criterion of the isolated system based on the concept of entransy, $\mathrm{d} G=0$, can describe the thermal equilibrium state effectively.

Let us consider another closed system shown in Figure 8 whose volume $V$ and environment temperature $T_{\mathrm{e}}$ are given. The environment can be treated as an infinitely great system. The mass, heat capacity and initial temperature of the closed system are $m, c$ and $T_{0}$ respectively. The heat transfer coefficient between the closed system and the environment is $h$, and the heat transfer area is $A$. The control equation of the heat transfer can then be expressed as

$$
c m \frac{\partial T}{\partial \tau}=h A\left(T_{\mathrm{e}}-T\right)
$$

where $T$ is the temperature of the closed system.

Letting $c=386 \mathrm{~J} \mathrm{~kg}^{-1} \mathrm{~K}^{-1}, m=1 \mathrm{~kg}, T_{\mathrm{e}}=300 \mathrm{~K}, T_{0}=280$ $\mathrm{K}$ and $h A=5 \mathrm{~W} \mathrm{~K}^{-1}$, the temperature of the closed system can

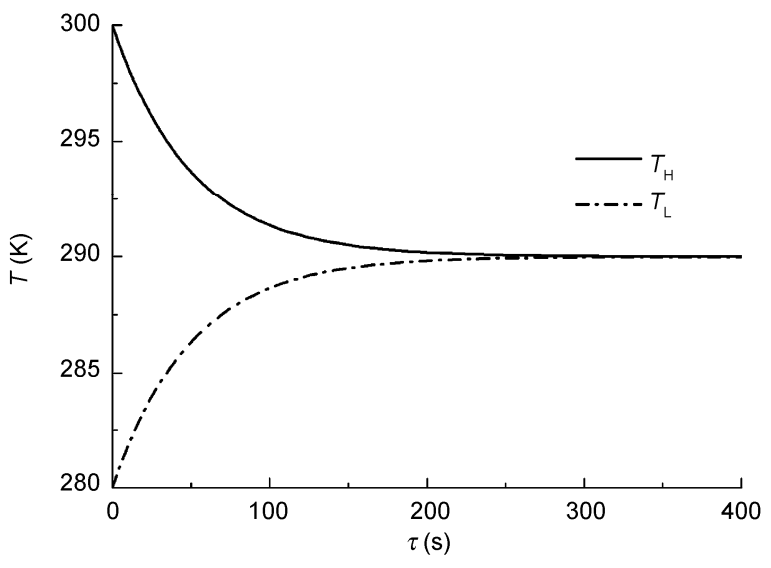

Figure 6 Temperature changes of the two bodies.

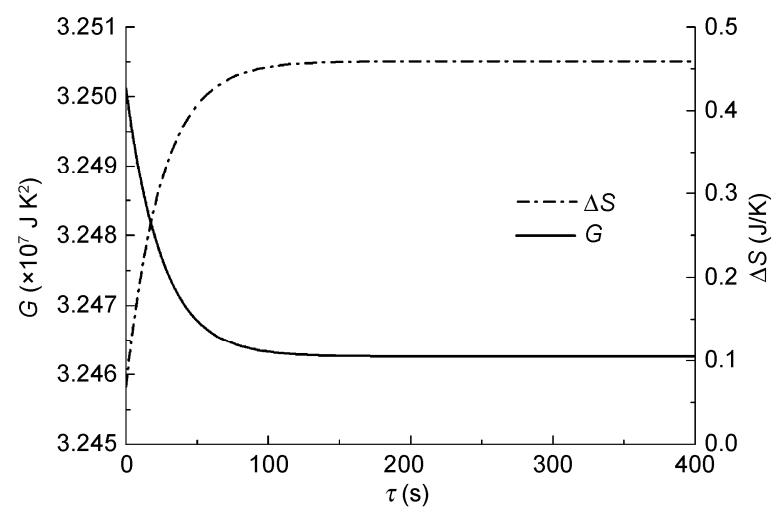

Figure 7 Entropy variation and change in entransy of the isolated system with time.

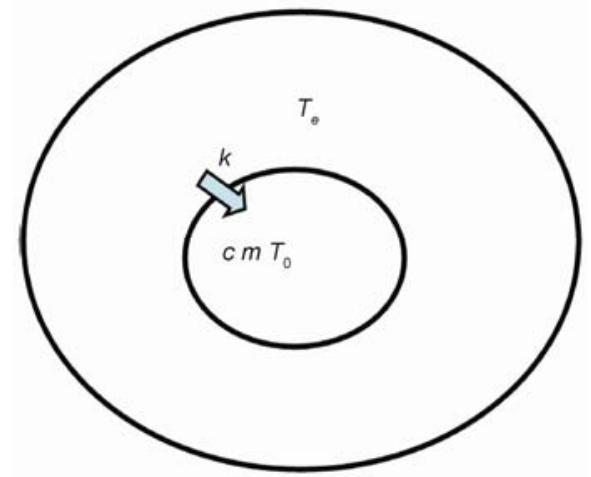

Figure 8 Heat transfer process in a simple closed system.

be determined using eq. (57), as well as the entropy increase and free entransy. The results are shown in Figure 9, where the entransy is calculated with eq. (2) while the entropy change is calculated with eq. (1).

It is seen that the temperature of the closed system increases gradually, and its asymptotic value is $300 \mathrm{~K}$, which is the temperature of the environment and also the thermal equilibrium temperature of the closed system. At the same time, the entropy of the isolated system, which consists of the closed system and the environment, increases gradually

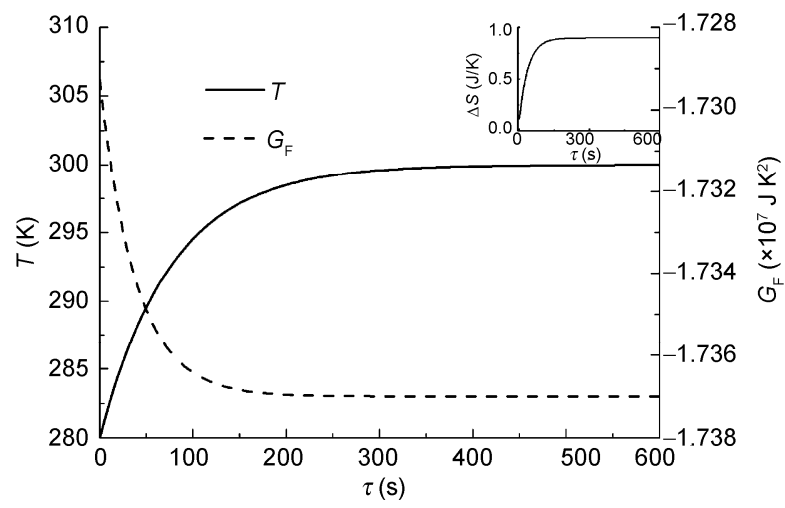

Figure 9 Variations in the temperature and free entransy of the closed system and the entropy change during the heat transfer. 
and has an asymptotic value, while the free entransy of the closed system decreases gradually and also has an asymptotic value. These variations demonstrate that both entropy and entransy can give the evolution direction of the heat transfer in the closed system. In addition, the minimum free entransy principle also describes the thermal equilibrium state effectively.

From the above discussion, it is found that entropy is not the only physical parameter that can be taken as the arrow of time in heat transfer processes; entransy is another. Like entropy, this new concept is also a measure of the irreversibility of heat transfer processes that do not involve work. At the same time, the thermal equilibrium criteria based on entransy also describe the thermal equilibrium state effectively.

\section{Conclusions}

On the basis of the concept of entransy and the first and second laws of thermodynamics, the entransy decrease principle for an isolated system is introduced in this paper. It is found that the entransy of an isolated system that does not involve work always decreases during heat transfer. This variation in entransy can be treated as the arrow of time in heat transfer and a measure of irreversibility. This principle can be taken as an expression of the second law of thermodynamics in heat transfer.

The thermal equilibrium criteria of the isolated system and the closed system are also developed. The criteria are referred to as the minimum entransy principle and the minimum free entransy principle, respectively. It is found that when an isolated system reaches thermal equilibrium, its entransy is a minimum. This is the minimum entransy principle. When a closed system reaches thermal equilibrium, its free entransy is also a minimum. This is the minimum free entransy principle. Therefore, like entropy, entransy can be regarded as the arrow of time in heat transfer processes and be used to describe the thermal equilibrium state of the isolated system and the closed system not involving work.

This work was supported by Tsinghua University Initiative Scientific Research Program.

1 Zhao K H, Luo W Y. Thermotics (in Chinese). Beijing: Higher Education Press, 2002. 1-222

2 Feng D, Feng S T. The World of Entropy (in Chinese). Beijing: Science Press, 2008. 104-160

3 Onsager L. Reciprocal relations in irreversible process II. Phys Rev, 1931, 38: 2265-2279

4 Onsager L, Machlup S. Fluctuations and irreversible processes. Phys Rev, 1953, 91: 1505-1512

5 Prigogine I. Introduction to Thermodynamics of Irreversible Processes. 3rd ed. New York: John Wiley \& Sons, 1967. 76-77

6 Bejan A. Entropy Generation Through Heat and Fluid Flow. New York: John Wiley \& Sons Inc, 1982. 119-134
7 Bejan A. Entropy Generation Minimization. Florida: CRC Press, 1996. 47-112

8 Bejan A. A study of entropy generation in fundamental convective heat transfer. ASME J Heat Transfer, 1979, 101: 718-725

9 Bejan A. Second-law analysis in heat transfer and thermal design. Adv Heat Transfer, 1982, 15: 1-58

10 Poulikakos D, Bejan A. Fin geometry for minimum entropy generation in forced convection. ASME J Heat Transfer, 1982, 104: 616-623

11 Erek A, Dincer I. An approach to entropy analysis of a latent heat storage module. Int J Thermal Sci, 2008, 47: 1077-1085

12 Shah R K, Skiepko T. Entropy generation extrema and their relationship with heat exchanger effectiveness-Number of transfer unit behavior for complex flow arrangements. J Heat Transfer, 2004, 126: 994-1002

13 Guo Z Y, Zhu H Y, Liang X G. Entransy-A physical quantity describing heat transfer ability. Int J Heat Mass Transfer, 2007, 50: 2545-2556

14 Guo Z Y, Cheng X G, Xia Z Z. Least dissipation principle of heat transport potential capacity and its application in heat conduction optimization. Chinese Sci Bull, 2003, 48: 406-410

15 Cheng X G, Li Z X, Guo Z Y. Heat conduction optimization based on least dissipation principle of heat transport potential capacity (in Chinese). J Eng Thermophys, 2003, 24: 94-96

16 Cheng X G. Entransy and its application in heat transfer optimization (in Chinese). Dissertation for Doctoral Degree. Beijing: Tsinghua University, 2004. 39-44

17 Zhu H Y. The minimum thermal resistance principle based on entransy dissipation. Dissertation for the Doctoral Degree. Beijing: Tsinghua University, 2007

18 Zhu H Y, Chen Z J, Guo Z Y. Thermal electric analogy experimental research for the extremum principle of entransy dissipation (in Chinese). Prog Nat Sci, 2007, 17: 1692-1698

19 Chen L G, Wei S H, Sun F R. Constructal entransy dissipation minimization for 'volume-point' heat conduction. J Phys D: Appl Phys, 2008, 41: 195506

20 Wei S H, Chen L G, Sun F R. "Volume-point" heat conduction constructal optimization with entransy dissipation minimization objective based on rectangular element. Sci China Ser E-Tech Sci, 2008, 51: 1283-1295

21 Cheng X T, Xu X H, Liang X G. Homogenization of temperature field and temperature gradient field. Sci China Ser E-Tech Sci, 2009, 52: 2937-2942

22 Chen Q, Ren J X. Generalized thermal resistance for convective heat transfer and its relation to entransy dissipation. Chinese Sci Bull, 2008, 53: 3753-3761

23 Wu J, Cheng X G, Meng J A, et al. Potential capacity dissipation extremum and entropy generation minimization in laminar convective heat transfer (in Chinese). J Eng Thermophys, 2006, 27: 100-102

24 Wu J, Liang X G. Application of entransy dissipation extremum principle in radiative heat transfer optimization. Sci China Ser E-Tech Sci, 2008, 51: 1306-1314

25 Cheng X T, Liang X G. Entransy flux of thermal radiation and its application to enclosures with opaque surfaces. Int J Heat Mass Transfer, 2011, 54: 269-278

26 Xia S J, Chen L G, Sun F R. Optimization for entransy dissipation minimization in heat exchanger. Chinese Sci Bull, 2009, 54: 3572-3578

27 Liu X B, Meng J A, Guo Z Y. Thermal resistance analysis for the heat exchangers based on entransy dissipation. Prog Nat Sci, 2008, 18: $1186-1190$

28 Liu X B, Meng J A, Guo Z Y. Entropy generation extremum and entransy dissipation extremum for heat exchanger optimization. Chinese Sci Bull, 2009, 54: 943-947

29 Liu X B, Guo Z Y. A novel method for heat exchanger analysis. Acta Phys Sin, 2009, 58: 4766-4771

30 Han G Z, Guo Z Y. Physical mechanism of heat conduction ability dissipation and its analytical expression (in Chinese). Proc CSEE, 2007, 27: $98-102$

Open Access This article is distributed under the terms of the Creative Commons Attribution License which permits any use, distribution, and reproduction in any medium, provided the original author(s) and source are credited. 